\title{
Long-term efficacy and safety of the Dumon stent for benign tracheal stenosis: a meta-analysis
}

\author{
Di-Fei Chen, Yu Chen, Chang-Hao Zhong, Xiao-Bo Chen, Shi-Yue Li
}

Department of Pulmonary and Critical Care Medicine, National Center for Respiratory Medicine, State Key Laboratory of Respiratory Disease, The First Affiliated Hospital of Guangzhou Medical University, National Clinical Research Center for Respiratory, Guangzhou Institute of Respiratory Health, Guangzhou, China

Contributions: (I) Conception and design: DF Chen, Y Chen, SY Li; (II) Administrative support: DF Chen, Y Chen, CH Zhong, SY Li; (III) Provision of study materials or patients: DF Chen, Y Chen, CH Zhong, XB Chen; (IV) Collection and assembly of data: DF Chen, Y Chen, CH Zhong, XB Chen; (V) Data analysis and interpretation: DF Chen, Y Chen; (VI) Manuscript writing: All authors; (VII) Final approval of manuscript: All authors. Correspondence to: Shi-Yue Li. Department of Respiratory, State Key Laboratory of Pulmonary and Critical Care Medicine, The First Affiliated Hospital of Guangzhou Medical University, National Clinical Research Center for Respiratory, Guangzhou Institute of Respiratory Health, 151 Yanjiang Road, Guangzhou 510120, China. Email: lishiyue@188.com.

\begin{abstract}
Background: The short-term efficacy of Dumon stent has been well demonstrated. Across years, however, due to insufficient sample size and absent of the randomized controlled trial, no reliable conclusion could be reach for Dumon stent's long-term efficacy. So, we conducted the first meta-analysis to evaluate the longterm efficacy and safety of the Dumon stent for benign tracheal stenosis.

Methods: Data on related trials were obtained by doing a literature search in PubMed, Web of Science and Cochrane Library. Random-effect and fixed-effect models were used to calculate the efficacy and incidence of complication of Dumon stent placement.

Results: A total of 395 patients from 8 studies were included in this study, revealing that the stability rate was $41.12 \%$ (95\% CI, 34.85-48.52\%) of Dumon stenting. Further, a curative rate of 40.74\% (95\% CI, $34.92-47.53 \%$ ), and efficacy of $75.49 \%$ (95\% CI, 70.89-80.39\%) were obtained from this study. Analysis of the incidence of complications indicated $25.04 \%$ of migration (95\% CI, 17.52-35.79\%), 15.66\% granulation (95\% CI, 9.39-26.11\%) and $23.82 \%$ of mucus retention $23.82 \%$ (95\% CI, $13.77-41.20 \%$ ).

Conclusions: Dumon stent has a moderate efficacy for treating benign tracheal stenosis with approximately $20 \%$ incidence of complication, regular bronchoscopy follow-up should be conducted. Thus, further research is required to modified Dumon stenting.
\end{abstract}

Keywords: Silicone stent; bronchoscopy; tracheal stenosis; meta-analysis

Submitted Jun 29, 2020. Accepted for publication Nov 05, 2020.

doi: $10.21037 /$ jtd-20-2327

View this article at: http://dx.doi.org/10.21037/jtd-20-2327

\section{Introduction}

Benign tracheobronchial stenosis which refers to abnormal narrowing of trachea and bronchus, is commonly caused by tracheal intubation, prior tracheotomy, tuberculosis, granulomatosis with polyangiitis and idiopathic strictures. Symptoms of tracheobronchial stenosis could develop immediately after extubation or progress slowly over 50\% airway obstruction occurs. The main symptoms of tracheal stenosis include stridor, dyspnea, and wheezing. Notably, some patients may present with lung function decline even though the respiratory symptoms absent (1).

Currently, surgery (tracheal resection and end-to-end anastomosis) serve as the most effective treatment for benign tracheal stenosis. A previous study reported over $90 \%$ success rate of surgery for treating airway stenosis, with only $10 \%$ complications occurred (2). However, less than $10 \%$ patients with tracheal stenosis are operable and 
resectable (3), since the surgery treatment has been limited by the high risks associated with anaesthesia and surgery, the length of the tracheal resection and the extent of the lesion. In addition, surgery-related deaths could increase thirteen-fold for patients with risk factors for anastomotic complications (diabetes, long-segment stenosis, previous tracheal surgery, etc.) (4). Thus, timely interventional bronchoscopy might contribute to the preferred approach for patients with inoperable stenosis, hyoxemia or relatedrespiratory failure (5).

Interventional bronchoscopy, present fewer complications compared with surgery, are more conducive to treat with elderly or patients with severe tracheal stenosis. Interventional managements include balloon dilatation, laser-assisted ablation, cryotherapy, and placement of stents, all of which could be applied alone or in combination (6). However, bronchoscopy has been reportedly induced airway injury, related granulation tissue and fibrous scar after interventional treatment (7).

Airway stenting is commonly used for maintaining luminal patency and providing structural support. Currently, the use of metallic stents in benign airway stenosis is controversial for its long-term severe complications., Furthermore, it has been a challenge to remove the prolonged placed metal stents from the airway, thus, FDA warned against the use of metallic stents for treating benign airway obstruction (8). Silicone stents, characterized by superior tolerance and less complications, display a good efficacy in patients with benign airway narrowing. Dumon silicone stent which was invented by JF Dumon in the 1980s, has a hollow and cylindrical shape, with external studs to prevent migration. Dumon stent is the most widely used silicone stent and considered as 'gold standard' for inoperable benign tracheal stenosis (9).

A previous study reported over $90 \%$ of patients who underwent silicone stent placement had rapid alleviation from dyspnea (10). However, assessing the long-term efficacy of silicone stent, includes stability rate, curative rates and incidence of restenosis might conducive to assess whether silicone stents could be used for treating benign tracheal stenosis. Whereas, the randomized controlled trials, prospective or retrospective studies on silicone stents with large sample sizes are currently absent. Here, we conducted a meta-analysis of retrospective studies to explore the longterm efficacy and safety of the Dumon stent as a treatment option for benign tracheal stenosis. We present the following article in accordance with the PRISMA reporting checklist (available at http://dx.doi.org/10.21037/jtd-20-2327).

\section{Methods}

Cochrane Handbook (11) and the PRISMA guidelines were used to guide the designing this study (12).

\section{Inclusion criteria}

Studies that satisfied the following criteria were included in this meta-analysis; (I) studies that included patients diagnosed with benign airway stenosis using bronchoscopy; (II) studies that included patients underwent silicone stent replacement; (III) studies where Dumon silicone stents were used; (IV) studies that included data on stent removal, stent stability, restenosis, or complications related to stent placement.

\section{Exclusion criteria}

Studies that only included malignant airway stenosis or those included cases with benign and malignant stenosis were excluded. Furthermore, we excluded the studies that investigated the effects of metallic stents. In addition, case reports and review articles were excluded. Articles written in non-English languages were not included in this study.

\section{Search strategy}

We conducted a literature search on Cochrane Library, PubMed (from 1981 to October 2018), the Web of Science (from 1981 to October 2018), and Google Scholar databases. The search algorithm was set as follows: ((benign AND ("tracheal stenosis"[MeSH Terms] OR ("tracheal" AND "stenosis") OR "tracheal stenosis")) OR (airway AND ("constriction, pathologic" [MeSH Terms] OR ("constriction" AND "pathologic") OR "pathologic constriction" OR "stenosis"))) AND ((Dumon AND ("stents"[MeSH Terms] OR "stents" OR "stent")) OR (("silicones"[MeSH Terms] OR "silicones" OR "silicone") AND ("stents"[MeSH Terms] OR "stents" OR "stent"))).

\section{Data retrieval}

All data were independently retrieved by two investigators (DC and YC). Disagreements were resolved by discussions between the two reviewers and an independent expert. Although some studies did not contain all required information, the following information were extracted from each study: first author, year of publication, number 
of patients, mean duration of stent placement, pathogeny of stenosis, treatment events including stent removal, stent stability, restenosis, and complications.

\section{Quality assessment}

The risk of bias was assessed by using the NewcastleOttawa scale (13). The overall score for each article was set at 9:4 for selection, 2 for comparability, and 3 for outcomes. Final score of 1-3, 4-6 and over 7-9, were defined as of low, moderate, and high quality respectively.

\section{Data synthesis}

All included studies were placed into three subgroups: $<6$, $6-12$, and $>12$ months groups based on the mean duration of Dumon stenting. These subgroups were used to assess the relationship between duration of stent application and efficacy. Further, we divided the study population into two groups, the palliative group and the treatment group, according to the aims of stent placement. The treatment group was used to study the long-term efficacy, while all the study population was used for analyzing the incidence of complications. Four indexes were chosen to evaluate the efficacy of Dumon stent for benign tracheal stenosis; (I) the curative rates (defined as the number of patients who had their stents successfully removed without symptomatic restenosis in 1 year); (II) the stability rates (defined as the number of patients who maintained stable stent placement); (III) the effective rates (defined as the sum of curative and stability rates added together) and (IV) the restenosis rates.

\section{Statistical analysis}

The total percentage for each event and number of cases in each study were obtained. The pooled effect sizes (ES), which denoted median "rates" were calculated and 95\% confidence intervals ( $95 \% \mathrm{CI}$ ), were used. Cochran's $\chi^{2}$ test and $\mathrm{I}^{2}$ were used to evaluate the heterogeneity of effect estimates; $\mathrm{P}<0.05$ or $\mathrm{I}^{2}>50 \%$ was considered statistically significant. A fixed-effects model was used except for cases had no significant heterogeneity. Studies with zero events were handled as described by Bradburn et al. (14) Publication bias was evaluated using the Egger linear test and a funnel plot was generated. All statistical analyses were conducted with $\mathrm{R}$ version 3.5.1.

\section{Results}

\section{Study characteristics}

We identified a total of 253 records from literature. Two hundred and twenty-nine out of 253 studies were excluded according to the inclusion and exclusion criteria. After reviewing the full text of the remaining 24 studies, 16 were excluded (Figure 1). A total of 8 retrospective studies comprising 395 patients met the inclusion criteria of this study (7,15-21). The characteristics of included studies were shown in Table 1 . The sample sizes of the 8 studies ranged from 13 to 125 . Most of the patients in these studies were young or middle-aged (mean age was 29-59 years). All studies focused on the treatment of benign airway stenosis patients using Dumon stent. Additionally, all 8 articles were single-arm studies, as shown by analysis using the Newcastle-Ottawa scale. Furthermore, 2 studies were of low quality while the remaining 6 were of moderate quality.

\section{Efficacy of Dumon stenting}

Analysis using the fixed effects models showed $40.74 \%$ curative rates $\left(\mathrm{I}^{2}=40.1 \%, \mathrm{P}=0.12,95 \% \mathrm{CI}, 34.92-47.53 \%\right)$, and the fixed effects model calculations showed $41.12 \%$ stability rate of Dumon stent in benign airway stenosis $\left(\mathrm{I}^{2}=37.9 \%, \mathrm{P}=0.14,95 \% \mathrm{CI}, 34.85-48.52 \%\right)$. In addition, effective rate of the stent approach as calculated by fixed effects model was $75.49 \%\left(\mathrm{I}^{2}=37.9 \%, \mathrm{P}=0.11,95 \% \mathrm{CI}\right.$, $70.89-80.39 \%$ ). The restenosis rate of $31.53 \%$ (95\% CI, $26.79-37.14 \%)$ was obtained using the random effects model (Figure 2).

\section{Safety of Dumon stenting}

In this study, safety events considered in this study included stent migration, granulation, and mucus retention. The rates of stent migration, granulation, and mucus retention were calculated using the random effects model for high heterogeneity; The stent migration rate of $25.04 \%$ (95\% CI, $17.52-35.79 \%$ ), while $15.66 \%$ of granulation rate was (95\% CI, 9.39-26.11\%), and $23.82 \%$ mucus retention rate (95\% CI, 13.77-41.20\%) were obtained (Figure 3).

\section{Subgroup analysis}

Subgroup analysis for stent duration was conducted using efficacy indexes. However, significant differences 


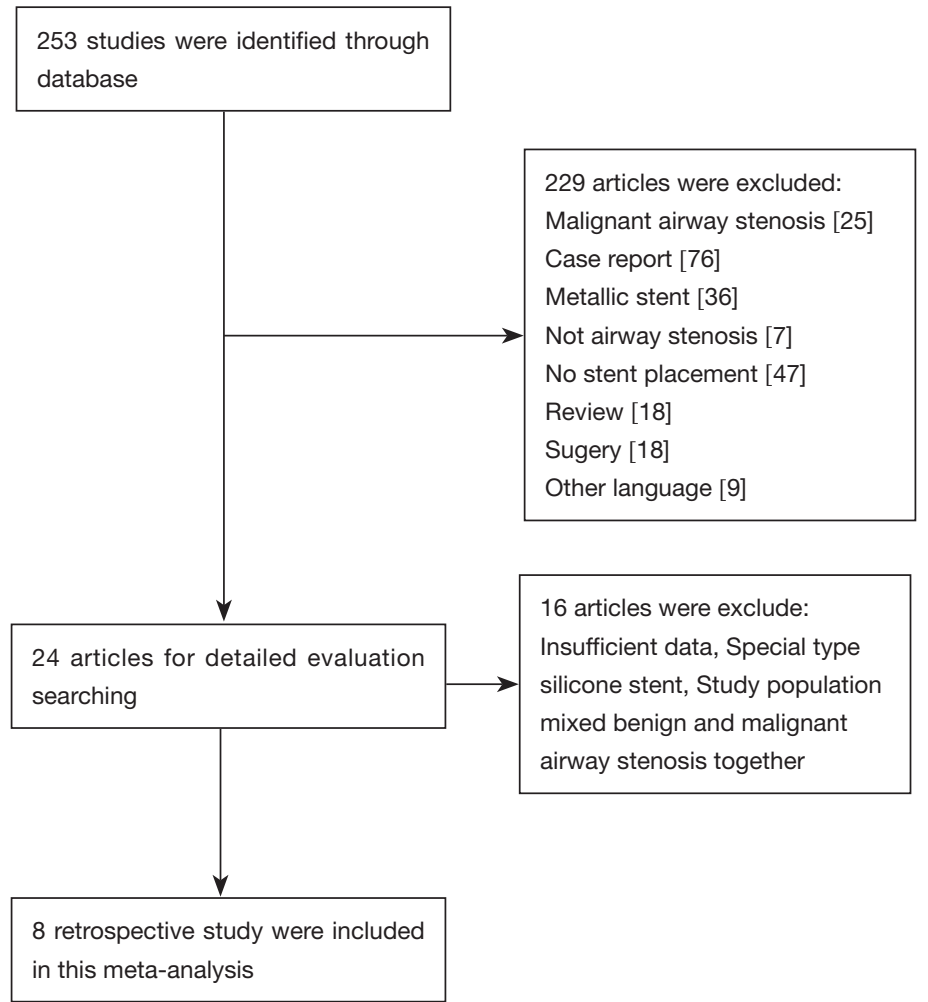

Figure 1 Flowchart demonstrating inclusion and exclusion criteria.

Table 1 Baseline characteristics of eight trials included for analysis

\begin{tabular}{|c|c|c|c|c|c|c|c|}
\hline Author & Pathogeny of stenosis & Type of stent & Mean age & Cases & $\begin{array}{l}\text { Treatment } \\
\text { group }\end{array}$ & $\begin{array}{l}\text { Mean duration of } \\
\text { stent (mouths) }\end{array}$ & Quality \\
\hline Martinez (1996) & $\begin{array}{l}\text { Postoperative stenosis, } \\
\text { postintubation stenosis }\end{array}$ & Dumon & 52 & 63 & 48 & 12.1 & Moderate \\
\hline Vergnon (2000) & $\begin{array}{l}\text { Postoperative stenosis, } \\
\text { postintubation stenosis }\end{array}$ & Dumon & 39.5 & 13 & 13 & 19.6 & Low \\
\hline Cavaliere (2007) & Postintubation stenosis & Dumon & 50 & 48 & 47 & 11.6 & Moderate \\
\hline Gallucio (2009) & $\begin{array}{l}\text { Postoperative stenosis, } \\
\text { postintubation stenosis }\end{array}$ & Dumon & 29 & 48 & 39 & 18 & Moderate \\
\hline Jeong (2012) & Postoperative stenosis & Dumon & 59 & 19 & 19 & 7 & Low \\
\hline Karush (2017) & $\begin{array}{l}\text { Postoperative stenosis, } \\
\text { postintubation stenosis }\end{array}$ & Dumon & 54 & 40 & 36 & 3.03 & Moderate \\
\hline
\end{tabular}




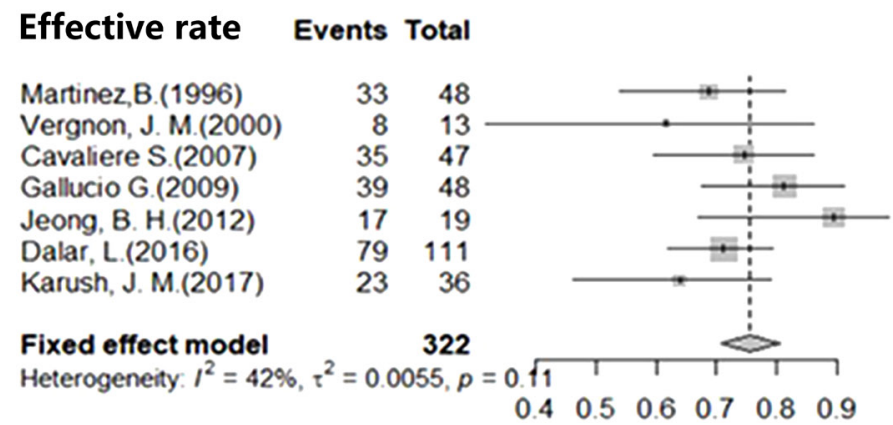

\section{Curative rate Events Total}

Martinez,B.(1996)
Vergnon, J.M.(2000)
Ryu, Y. J.(2006)
Cavaliere S.(2007)
Gallucio G.(2009)
Jeong, B. H.(2012)
Karush, J. M.(2017)

Fixed effect model Heterogeneity: $I^{2}=40 \%, \tau^{2}=0.0311, p=\begin{array}{lllllll}0.12 & 1 & 1 & 1 & \\ 0.1 & 0.2 & 0.3 & 0.4 & 0.5 & 0.6\end{array}$

\section{Proportion $\quad 95 \%-\mathrm{Cl}$ Weight}

$0.69[0.54 ; 0.81] \quad 10.9 \%$

$0.62[0.32 ; 0.86] \quad 2.1 \%$

$0.74[0.60 ; 0.86] \quad 14.1 \%$

$0.81[0.67 ; 0.91] 21.4 \%$

$0.89[0.67 ; 0.99] 16.6 \%$

$0.71[0.62 ; 0.79] \quad 28.2 \%$

$0.64[0.46 ; 0.79] \quad 6.6 \%$

$0.75[0.71 ; 0.80] 100.0 \%$
Proportion $\quad 95 \%-\mathrm{Cl}$ Weight

$\begin{array}{llr}0.35 & {[0.22 ; 0.51]} & 16.3 \% \\ 0.31 & {[0.09 ; 0.61]} & 3.6 \% \\ 0.49 & {[0.32 ; 0.65]} & 22.9 \% \\ 0.49 & {[0.34 ; 0.64]} & 27.9 \% \\ 0.40 & {[0.26 ; 0.55]} & 19.5 \% \\ 0.32 & {[0.13 ; 0.57]} & 5.4 \% \\ 0.17 & {[0.06 ; 0.33]} & 4.5 \%\end{array}$

$0.41[0.35 ; 0.48] 100.0 \%$

\section{Stability rate}

Martinez,B.(1996)

Vergnon, J. M.(2000)

Cavaliere S.(2007)

Gallucio G.(2009)

Jeong, B. H.(2012)

Karush, J. M.(2017)

Fixed effect model

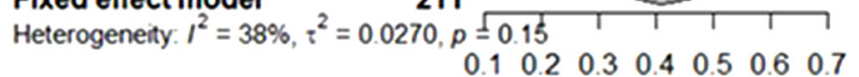

\section{Events Total}

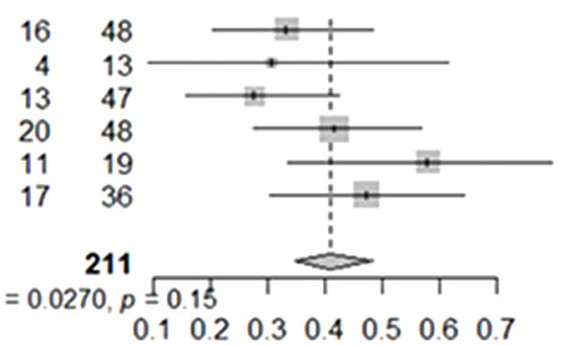

Proportion $\quad 95 \%-\mathrm{Cl}$ Weight

$\begin{array}{llr}0.33 & {[0.20 ; 0.48]} & 17.1 \% \\ 0.31 & {[0.09 ; 0.61]} & 4.1 \% \\ 0.28 & {[0.16 ; 0.43]} & 12.8 \% \\ 0.42 & {[0.28 ; 0.57]} & 24.4 \% \\ 0.58 & {[0.33 ; 0.80]} & 18.6 \% \\ 0.47[0.30 ; 0.65] & 22.9 \% \\ 0.41 & {[0.35 ; 0.49]} & 100.0 \%\end{array}$
$0.31 \quad[0.09 ; 0.61] \quad 4.1 \%$ $0.28[0.16 ; 0.43] \quad 12.8 \%$ $0.42[0.28 ; 0.57] \quad 24.4 \%$ $0.58 \quad[0.33 ; 0.80] \quad 18.6 \%$ $0.41[0.35 ; 0.49] 100.0 \%$

\section{Restenosis rate}

Martinez,B.(1996)

Vergnon, J. M. (2000)

Ryu, Y. J.(2006)

Cavaliere S.(2007)

Gallucio G.(2009)

Jeong, B. H.(2012)

Dalar, L. (2016)

Karush, J. M.(2017)

Random effects model
Heterogeneity. $I^{2}=72 \%, \tau^{2}=0.1596, \rho<0.0$

Events Total

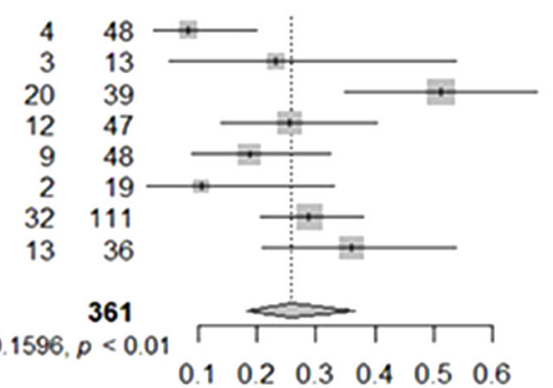

Proportion $\quad 95 \%-\mathrm{Cl}$ Weight

$0.08 \quad[0.02 ; 0.20] \quad 8.3 \%$

$0.23[0.05 ; 0.54] \quad 7.8 \%$

$0.51 \quad[0.35 ; 0.68] \quad 17.6 \%$

$0.26[0.14 ; 0.40] \quad 14.6 \%$

$0.19[0.09 ; 0.33] \quad 13.0 \%$

$0.11[0.01 ; 0.33] \quad 5.3 \%$

$0.29[0.21 ; 0.38] \quad 17.8 \%$

$0.36[0.21 ; 0.54] \quad 15.5 \%$

$0.26[0.18 ; 0.37] 100.0 \%$

Figure 2 Forest plot for the meta-analysis of efficacy indexes. CI, confidence interval. 

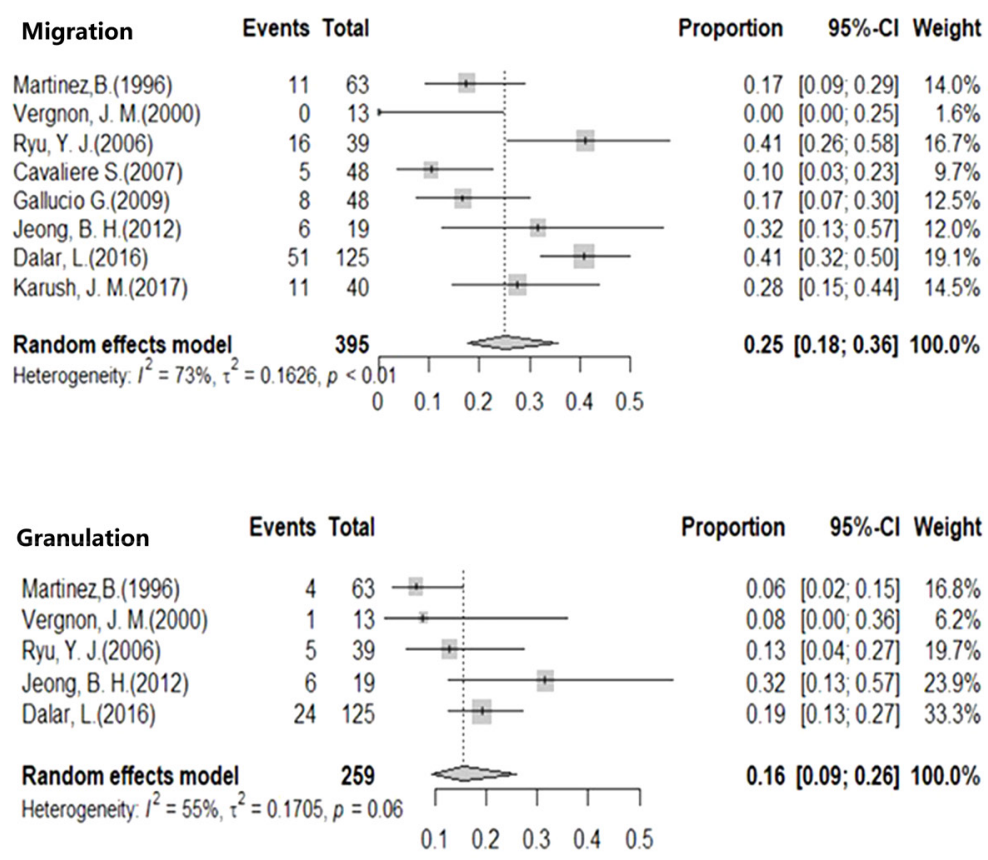

Proportion $\quad 95 \% \cdot \mathrm{Cl}$ Weight

$0.06[0.02 ; 0.15] \quad 16.8 \%$

$0.08[0.00 ; 0.36] \quad 6.2 \%$

$0.13[0.04,0.27] \quad 19.7 \%$

$0.32[0.13 ; 0.57] \quad 23.9 \%$

$0.19[0.13 ; 0.27] \quad 33.3 \%$

$0.16[0.09 ; 0.26] 100.0 \%$

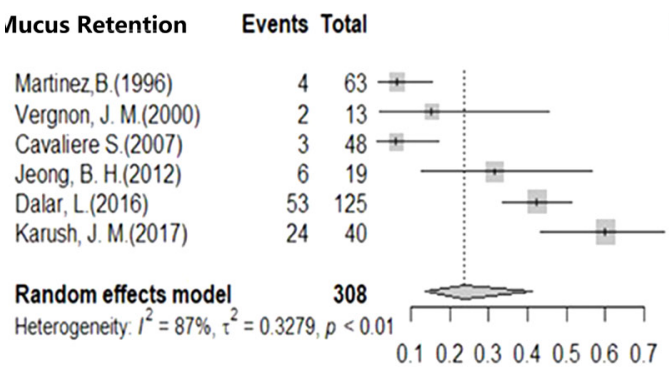

Proportion $95 \% \cdot \mathrm{Cl}$ Weight

$0.06[0.02 ; 0.15] \quad 13.9 \%$

$0.15[0.02 ; 0.45] \quad 10.4 \%$

$0.06[0.01 ; 0.17] \quad 12.2 \%$

$0.32[0.13 ; 0.57] \quad 17.7 \%$

$0.42[0.34 ; 0.52] \quad 23.1 \%$

$0.60[0.43,0.75] 22.7 \%$

$0.24[0.14 ; 0.41] 100.0 \%$

Figure 3 Forest plot for the meta-analysis of safety events. CI, confidence interval.

between subgroups were only observed for the removal group $(\mathrm{P}=0.0402)$. The curative rate in the $<6$-month group was $16.67 \%$ (95\% CI, $8.03-34.60 \%$ ), $43.66 \%$ in the 6-12-month group (95\% CI, 29.95-63.64\%), and 40.91\% in the $>12$-month group (95\% CI, 33.65-49.74\%) (Figure 4). Post hoc test, showed significant differences between the $<6$-month group and the other two groups $(\mathrm{P}<0.05)$. There were no significant differences in efficacy rates between 6-12-month and >12-month groups (Figure S1).

\section{Publication bias}

A P value of 0.831 for the Egger linear test was obtained, therefore, no publication bias was not observed in this metaanalysis ( $t=0.22486$ ). Consistent with the result of Egger linear test results, a symmetrical funnel plot was obtained (Figure 5).

\section{Discussion}

Dumon stent has been used for the treating airway stenosis for about 40 years. However, the efficacy and related complications of Dumon stent has not been well elucidated by prospective clinical trials multicenter study. To our knowledge, this is the first meta-analysis study to include retrospective, single-arm, and single-center studies on use of Dumon stent for treating benign tracheal stenosis. The current study showed Dumon stent has $75.49 \%$ effective rate and approximately $20 \%$ incidence of complications (migration, granulation, and mucus retention) indicating that Dumon stent had moderate efficacy and safety for airway stenosis.

Previous studies reported that over $90 \%$ of patients experienced quick relief of dyspnea (10), rather than the 


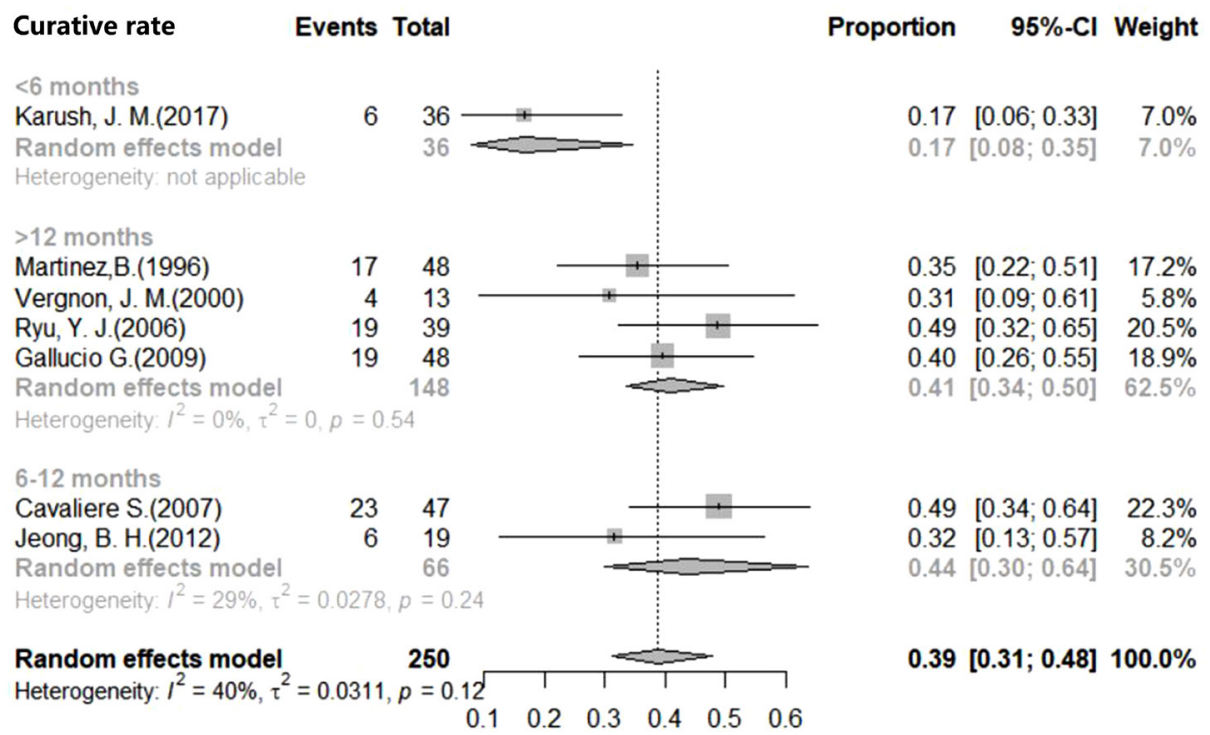

Figure 4 Forest plots for subgroup meta-analysis of curative rate based on median follow-up. CI, confidence interval.

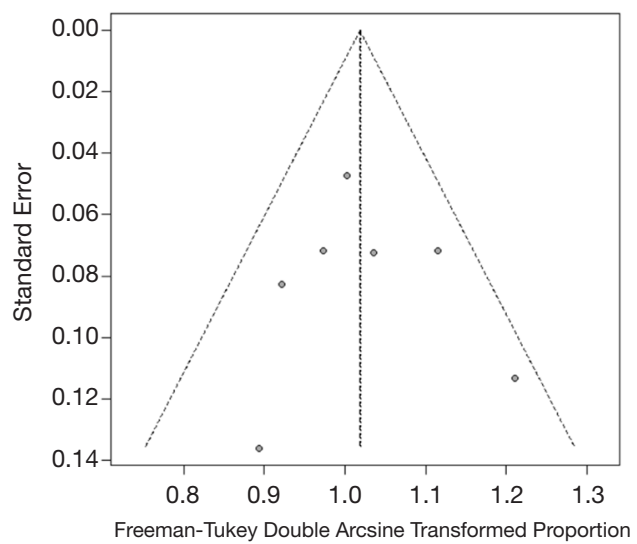

Figure 5 Funnel plot of studies included in this meta-analysis.

complete elimination of tracheal stenosis. In attempt to evaluate the placement of silicone stent, Borg scale, lung function, CT measurement of stenosis, curative rates (successful removal of the stent) and stability rates (stenosis remaining stable after stent implantation) after stent placement (8). Curative rates and stability rates are summed up to serve as a long-term index denoted as effective rate to evaluate the overall performance of Dumon stenting. In this study, we employed the restenosis rate as a part of efficacy rate to evaluate of the treatment failure. In the current analysis, the stability rate was $41.12 \%$ (95\% CI, 34.85-48.52\%), the curative rate $40.74 \%$ (95\% CI, $34.92-47.53 \%$ ), the effective rate $75.49 \%$ (95\% CI,
$70.89-80.39 \%)$ and the restenosis rate $31.53 \%$ (95\% CI, 26.79-37.14\%). These results indicated that Dumon stent was effective in over $75 \%$ patients with tracheal stenosis. However, more than $40 \%$ of patients had to maintain stent placement to ensure the airway luminal stability, and stent therapy is not effective in approximately $30 \%$ of patients.

Analysis of duration of stent placement showed no significant differences for effective rates, stability rates, and restenosis rates in all three subgroup. Moreover, the effective rates, stability rates and restenosis rates showed no correlation with the duration of stent use. However, the curative rates were significantly lower in $<6$-month group compared with those in 6-12-month and $>12$-month group. These findings implied that untimingly early removal of stent should be avoided to ensure the stability of that stenosis lesion with stents. The appropriate timing of stent removal is not definite. Our results were compatible with those of Dumon's report that stents should be left in place for more than 6 months (22), which could significantly improve the success rate of stent removal without increasing the restenosis rate. Verma et al. explored the definite stent removal time by using computerized tomography (CT) in post-tuberculosis tracheobronchial stenosis (23). In this study, the length of air pockets (tracheobronchial air columns in the space between the outer surface of the stent and the adjacent airway wall) in CT was used as an indicator for stent removal. Pockets longer than 1 or $2 \mathrm{~cm}$ could be used to indicate a successful stent removal, with $84 \%$ 
sensitivity and $50 \%$ specificity.

The major complications of Dumon stent includes migration, granulation, and mucus retention $(10,24)$. Metaanalysis showed various incidences of complications from those reported in the previous studies, migration rate at $18.6 \%$, granulation rate at $17.2 \%$, mucus retention rate at $5.7 \%$ (22). In our study, migration rate was slightly higher $(25.04 \%)$ while the incidence of mucus retention was significantly higher $(23.82 \%)$ than the incidence reported than those reported in previous study (9). Silicone stent related complications are commonly occurred in patients with benign tracheal stenosis. Severe mucus retention induces asphyxia and requires immediate clearance or respiratory interventions to prevent suffocation. Extensive migration of stent near the vocal cord could cause immediate asphyxia. External fixation of the silicone stent is used to reduce the occurrence of migration, whereas, the curative effect is commonly limited by the complex technique and procedure $(25,26)$. The accurate assessment of stenosis lesion and precise measurement of lumen dimensions might be of great important to improve the efficacy of airway stenting. Regular bronchoscopy followup should be conducted after Dumon stenting for early identification and management of related complications.

This study had several limitations. Firstly, the definite timing for Dumon stent remove were absent. The current study indicated that Dumon stent should be implanted for at least 6 months before removal. Secondly, as the study population was small, the included patients could not grouped by etiology of benign tracheal stenosis. Shin et al. reported a clinical significant difference in efficacy between post-intubation tracheal stenosis (PITS) and post-tracheostomy tracheal stenosis (PTTS). Silicone stent removal was achieved in $46.2 \%$ of patients in the PITS group and in $33.0 \%$ of patients in the PTTS group $(\mathrm{P}=0.063)$ (27). Thirdly, we only studied Dumon stent which is the most common airway stent, rather than including the other types of silicone stents, such as natural, dynamic and polyflex. Moreover, the current study did not investigate the other non-surgical treatment (i.e., balloon-dilation or laser ablation) in pre-and postoperative care, which might affect the prognosis of stent treatment. Finally, this meta-analysis only included retrospective single-arm studies and we only included studies reported in English, it's worth noting that tuberculosis related airway stenosis, one of the most common type of benign tracheal stenosis, post-tuberculosis airway stenosis, is highly prevalent in Asia (28).
Recent studies have reported some novel designs of silicone stents. Oki et al. conducted a retrospective study on a novel silicone stent, designed to fit in the primary right carina for treating airway stenosis (29). Advances in Materialogy have increased interest in study of customized stents. Gildea et al. (30) reported the application of 3D patient-specific stent in the treatment of Wegener's granuloma. Drug-eluting stents have been designed for treating airway infections and granulation $(31,32)$.

\section{Conclusions}

Dumon stent has a moderate efficacy for treating benign tracheal stenosis with approximately $20 \%$ incidence of complication, regular bronchoscopy follow-up should be conducted. Multi-center, prospective clinical trials should be highlighted and performed for overall assessment of the silicone stent. Furthermore, novel designs, customize stent and tissue-engineering stent would be a crucial and promising in the development of airway stenting.

\section{Acknowledgments}

To memorize the founder silicone stent, inventor of Dumon stent, JF Dumon (1939-2020), R.I.P.

Funding: None.

\section{Footnote}

Reporting Checklist: The authors have completed the PRISMA reporting checklist. Available at http://dx.doi. org/10.21037/jtd-20-2327

Peer Review File: Available at http://dx.doi.org/10.21037/jtd20-2327

Conflicts of Interest: All authors have completed the ICMJE uniform disclosure form (available at http://dx.doi. org/10.21037/jtd-20-2327). The authors have no conflicts of interest to declare.

Ethical Statement: The authors are accountable for all aspects of the work in ensuring that questions related to the accuracy or integrity of any part of the work are appropriately investigated and resolved.

Open Access Statement: This is an Open Access article distributed in accordance with the Creative Commons Attribution-NonCommercial-NoDerivs 4.0 International 
License (CC BY-NC-ND 4.0), which permits the noncommercial replication and distribution of the article with the strict proviso that no changes or edits are made and the original work is properly cited (including links to both the formal publication through the relevant DOI and the license). See: https://creativecommons.org/licenses/by-nc-nd/4.0/.

\section{References}

1. Stoelben E, Koryllos A, Beckers F, et al. Benign stenosis of the trachea. Thorac Surg Clin 2014;24:59-65.

2. D'Andrilli A, Venuta F, Rendina EA. Subglottic tracheal stenosis. J Thorac Dis 2016;8:S140-7.

3. Nouraei SM, Middleton SE, Nouraei SA, et al. Management and prognosis of primary tracheal cancer: a national analysis. Laryngoscope 2014;124:145-50.

4. Auchincloss HG, Wright CD. Complications after tracheal resection and reconstruction: prevention and treatment. J Thorac Dis 2016;8:S160-7.

5. Ahn HY, Su Cho J, Kim YD, et al. Surgical outcomes of post intubational or post tracheostomy tracheal stenosis: report of 18 cases in single institution. Ann Thorac Cardiovasc Surg 2015;21:14-7.

6. Özdemir C, Kocatürk CI, Sökücü SN, et al. Endoscopic and Surgical Treatment of Benign Tracheal Stenosis: A Multidisciplinary Team Approach. Ann Thorac Cardiovasc Surg 2018;24:288-95.

7. Dalar L, Karasulu L, Abul Y, et al. Bronchoscopic Treatment in the Management of Benign Tracheal Stenosis: Choices for Simple and Complex Tracheal Stenosis. Ann Thorac Surg 2016;101:1310-7.

8. Ayub A, Al-Ayoubi AM, Bhora FY. Stents for airway strictures: selection and results. J Thorac Dis 2017;9:S116-21.

9. Lee P, Kupeli E, Mehta AC. Airway stents. Clin Chest Med 2010;31:141-50, Table of Contents.

10. Walser EM. Stent placement for tracheobronchial disease. Eur J Radiol 2005;55:321-30.

11. Higgins JPT, Thomas J, Chandler J, et al. Cochrane Handbook for Systematic Reviews of Interventions version 6.0 (updated July 2019). Cochrane, 2019. Available online: www.training.cochrane.org/handbook

12. Swartz MK. The PRISMA statement: a guideline for systematic reviews and meta-analyses. J Pediatr Health Care 2011;25:1-2.

13. Wells G, Shea B, O'Connell D, et al. The NewcastleOttawa Scale (NOS) for assessing the quality of nonrandomised studies in meta-analyses. 2007. Available online: http://www ohri ca/programs/clinical epidemiology/oxford htm

14. Bradburn MJ, Deeks JJ, Berlin JA, et al. Much ado about nothing: a comparison of the performance of meta-analytical methods with rare events. Stat Med 2007;26:53-77.

15. Martinez-Ballarin JI, Diaz-Jimenez JP, Castro MJ, et al. Silicone stents in the management of benign tracheobronchial stenoses. Tolerance and early results in 63 patients. Chest 1996;109:626-9.

16. Vergnon JM, Costes F, Polio JC. Efficacy and tolerance of a new silicone stent for the treatment of benign tracheal stenosis: preliminary results. Chest 2000;118:422-6.

17. Ryu YJ, Kim H, Yu CM, et al. Comparison of natural and Dumon airway stents for the management of benign tracheobronchial stenoses. Respirology 2006;11:748-54.

18. Cavaliere S, Bezzi M, Toninelli C, et al. Management of post-intubation tracheal stenoses using the endoscopic approach. Monaldi Arch Chest Dis 2007;67:73-80.

19. Galluccio G, Lucantoni G, Battistoni P, et al. Interventional endoscopy in the management of benign tracheal stenoses: definitive treatment at long-term follow-up. Eur J Cardiothorac Surg 2009;35:429-33; discussion 933-4.

20. Jeong BH, Um SW, Suh GY, et al. Results of interventional bronchoscopy in the management of postoperative tracheobronchial stenosis. J Thorac Cardiovasc Surg 2012;144:217-22.

21. Karush JM, Seder CW, Raman A, et al. Durability of Silicone Airway Stents in the Management of Benign Central Airway Obstruction. Lung 2017;195:601-6.

22. Lyamula EF, Magesa PM, Mhalu FS. Problems and solutions associated with transmission of HIV via donated blood in Tanzania. Afr J Health Sci 1996;3:6-10.

23. Verma A, Park HY, Lim SY, et al. Posttuberculosis tracheobronchial stenosis: use of CT to optimize the time of silicone stent removal. Radiology 2012;263:562-8.

24. Murgu SD, Colt HG. Complications of silicone stent insertion in patients with expiratory central airway collapse. Ann Thorac Surg 2007;84:1870-7.

25. Dhooria S, Agarwal R. External fixation of a subglottic tracheal silicone stent. Ann Am Thorac Soc 2014;11:467-8.

26. Majid A, Fernandez-Bussy S, Kent M, et al. External fixation of proximal tracheal airway stents: a modified technique. Ann Thorac Surg 2012;93:e167-9.

27. Shin B, Kim K, Jeong BH, et al. Clinical significance of differentiating post-intubation and post-tracheostomy tracheal stenosis. Respirology 2017;22:513-20.

28. Wan IY, Lee TW, Lam HC, et al. Tracheobronchial stenting for tuberculous airway stenosis. Chest 
2002;122:370-4.

29. Oki M, Saka H. New dedicated bifurcated silicone stent placement for stenosis around the primary right carina. Chest 2013;144:450-5.

30. Gildea TR, Young BP, Machuzak MS. Application of 3D Printing for Patient-Specific Silicone Stents: 1-Year Follow-Up on 2 Patients. Respiration 2018;96:488-94.

Cite this article as: Chen DF, Chen Y, Zhong CH, Chen XB, Li SY. Long-term efficacy and safety of the Dumon stent for benign tracheal stenosis: a meta-analysis. J Thorac Dis 2021;13(1):82-91. doi: 10.21037/jtd-20-2327
31. Duvvuri M, Motz K, Tsai HW, et al. Design of a Biocompatible Drug-Eluting Tracheal Stent in Mice with Laryngotracheal Stenosis. J Vis Exp 2020;(155):10.3791/60483.

32. Duvvuri M, Motz K, Murphy M, et al. Engineering an immunomodulatory drug-eluting stent to treat laryngotracheal stenosis. Biomater Sci 2019;7:1863-74. 


\section{Supplementary}

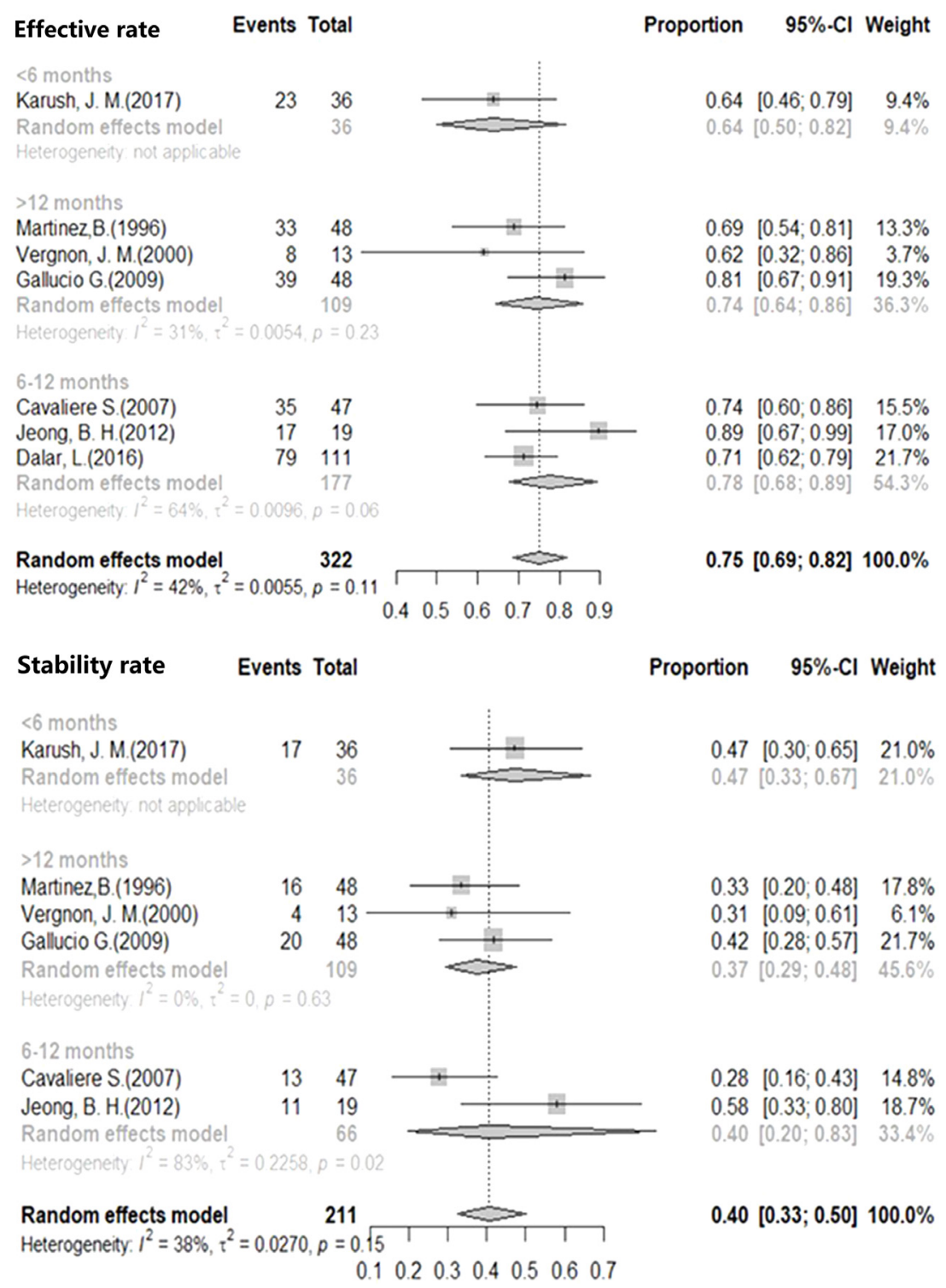

Figure S1 Forest plots for subgroup meta-analysis of effective rate and stability rate based on median follow-up. CI, confidence interval. 